\title{
129. The Solution of the Generalized Boussinesq's Problem for Elastic Foundation. I
}

\author{
By Bennosuke TANIMOTO \\ Faculty of Engineering, Shinshu University, Nagano, Japan \\ (Comm. by Y. Tanaka, M.J.A., Oct. 12, 1955)
}

Since the time of Boussinesq, theoretical basis for the problem of the safety of elastic foundation has been discussed and developed by various investigators, of which the works by Prof. Terazawa ${ }^{1)}$ and Love ${ }^{2)}$ are noteworthy. The former discussed it in detail when the loaded area is of circular form, and the latter gave the integration of Boussinesq's potentials when a uniform pressure extends over a rectangular area. But as is well known, the Boussinesq's potential method cannot be compatible with shearing forces at all, which would be of considerable importance especially in the case of soft foundation. In addition the Boussinesq's potentials are difficult to perform their integrations, and only the simplest case cited has been treated by Love. Numerical process of their integration is also almost impossible by ordinary methods of numerical integration, since integrands involved have an infinite number of singular points.

The boundary-value problem here treated is that, within a rectangular form of loaded area, any distributions of two kinds of shearing forces as well as of normal pressure are given on the semi-infinite elastic solid, provided these three kinds of external forces are expressible in terms of Fourier's integral in two dimensions.

The procedure of the calculation is for convenience due to a new set of functions which has been proposed by me and might be called stress-functions in three dimensions. ${ }^{3}$ The resulting solution is obtained in the forms of Fourier's integral, and the evaluation of the integrals was relied on the method of mechanical cubature ${ }^{4) 5}$ ) because of the difficulty in its analytical performance. As regards stresses there is no singularity in the integrands, and to secure first two or three significant figures in the numerical result is not so laborious. Displacements can also be integrated, in spite that each of integrands involved has one singularity at the origin of parametric coordinates.

Applications of the general solution will be given to several cases: (1) uniform pressure, (2) uniformly varying pressure, (3) uniform shearing force, (4) uniformly varying shearing force, etc. 
These solutions can at once be written down from the general solution.

The general boundary conditions with which we are now to deal are thus:

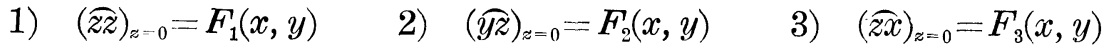
for $-a<x<a, \quad-b<y<b$.

4) These three functions entirely vanish, outside of the rectangular domain.

5) All the stresses vanish, when $x, y, z$ become indefinitely great.

Now Fourier's integral theorem gives

$$
F(x, y)=\frac{1}{\pi^{2}} \int_{0}^{\infty} d \alpha \int_{0}^{\infty} d \beta \int_{-\infty}^{\infty} \int_{-\infty}^{\infty} F(\xi, \eta) \cos \alpha(x-\xi) \cos \beta(y-\eta) d \xi d \eta,
$$

$F(x, y)$ representing one of $F_{1}(x, y), F_{2}(x, y)$, and $F_{3}(x, y)$, and hence the boundary conditions 1) -3 ) will require 12 typical solutions. thus: ${ }^{3)}$

The proposed procedures for three-dimensional elasticity are

$$
\begin{aligned}
& u=\frac{1}{2 \mu} \frac{\partial \phi}{\partial x}+\frac{1}{2 \mu}\left\{-\frac{\partial}{\partial x} \nabla^{2}+(1-\sigma)\left(\frac{\partial}{\partial y}+\frac{\partial}{\partial z}\right) \nabla^{2}\right\} x \\
& +\frac{1}{2 \mu}\left(\begin{array}{cc}
\partial \\
\partial y
\end{array}-\frac{\partial}{\partial z}\right) \psi \\
& \widehat{x x}=\frac{\partial^{2} \phi}{\partial x^{2}}+\left\{\left(\frac{\partial^{2}}{\partial y^{2}}+\begin{array}{c}
\partial^{2} \\
\partial z^{2}
\end{array}\right) \nabla^{2}--(1-\sigma) \frac{\partial^{2}}{\partial y \partial z} \nabla^{2}\right\} x \\
& +\left(\begin{array}{c}
\partial^{2} \\
\partial x \partial y
\end{array}-\frac{\partial^{2}}{\partial z \partial x}\right) \psi \\
& \widehat{y z}=\frac{\partial^{2} \phi}{\partial y \partial z}+\left\{-\frac{\partial^{2}}{\partial y \partial z} \nabla^{2}+\frac{1-\sigma}{2} \frac{\partial}{\partial x}\left(-\frac{\partial}{\partial x}+\frac{\partial}{\partial y}+\frac{\partial}{\partial z}\right) \nabla^{2}\right\} x \\
& +\frac{1}{2}\left(\begin{array}{c}
\partial^{2} \\
\partial x \partial y
\end{array}-\frac{\partial^{2}}{\partial y^{2}}+\frac{\partial^{2}}{\partial z^{2}}-\frac{\partial^{2}}{\partial z \partial x}\right) \psi, \ldots,
\end{aligned}
$$

where $\mu$ is the modulus of rigidity, and $\sigma$ Poisson's ratio for the material. Other components of displacement and stress are given by cyclical interchange of $x, y, z$. The functions $\phi, \chi$, and $\psi$ satisfy respectively the equations

$$
\nabla^{2} \phi=0, \quad \nabla^{4} \chi=0, \quad \text { and } \quad \nabla^{2} \psi=0,
$$

and $\quad \nabla^{2}=\frac{\partial^{2}}{\partial x^{2}}+\frac{\partial^{2}}{\partial y^{2}}+\frac{\partial^{2}}{\partial z^{2}}, \quad \nabla^{2}=\frac{\partial^{2}}{\partial y \partial z}+\frac{\partial^{2}}{\partial z \partial x}+\frac{\partial^{2}}{\partial x \partial y}$.

The $\phi$-function which is harmonic is, for the convenience of calculation, extracted from the $\chi$-function, and it has long since been known as 'displacement-potential.' ${ }^{6)}$

Typical solutions suitable for the present boundary-value problem are 


$$
\begin{aligned}
\phi= & \left(A_{1} \cos \alpha x \cos \beta y+A_{2} \cos \alpha x \sin \beta y\right. \\
& \left.+A_{3} \sin \alpha x \cos \beta y+A_{4} \sin \alpha x \sin \beta y\right) e^{-\gamma z}, \\
\psi= & \left(B_{1} \cos \alpha x \cos \beta y+B_{2} \cos \alpha x \sin \beta y\right. \\
& \left.+B_{3} \sin \alpha x \cos \beta y+B_{4} \sin \alpha x \sin \beta y\right) e^{-\gamma z}, \\
\chi= & \left(C_{1} \cos \alpha x \cos \beta y+C_{2} \cos \alpha x \sin \beta y\right. \\
& \left.+C_{3} \sin \alpha x \cos \beta y+C_{4} \sin \alpha x \sin \beta y\right) z e^{-\gamma z},
\end{aligned}
$$

where $A_{1}, A_{2}, \ldots C_{3}, C_{4}$ are constants to be determined; $\alpha, \beta$, and $\gamma$ being parameters provided $\alpha^{2}+\beta^{2}=\gamma^{2}$.

Stresses are calculated by substitution, and the foregoing boundary conditions afford a set of 12 equations whose unknowns are $A_{1}, A_{2}, \ldots$ $C_{3}, C_{4}$. These equations can be solved by the procedure that any one of 12 constants which come from Fourier's integral is survived and all the remaining constants are supposed to be zero, and that 12 sets of simultaneous equations thus formed are solved individually; each of them at once reduces to equations of three unknowns by simple transformations. Accordingly seeming difficulties in solving the enormous simultaneous equations could be resolved.

Thus the required general solution becomes as follows:

$$
\begin{aligned}
& \widehat{z z}=\frac{1}{\pi^{2}} \int_{0}^{\infty} d \alpha \int_{0}^{\infty} d \beta \int_{-\infty}^{\infty} \int_{-\infty}^{\infty}\left[(1+\gamma z) F_{1}(\xi, \eta) \cos \alpha(x-\xi) \cos \beta(y-\eta)\right. \\
& +\beta z F_{2}(\xi, \eta) \cos \alpha(x-\xi) \sin \beta(y-\eta) \\
& \left.+\alpha z F_{3}(\xi, \eta) \sin \alpha(x-\xi) \cos \beta(y-\eta)\right] e^{-\tau z} d \xi d \eta, \\
& \widehat{y z}=\frac{1}{\pi^{2}} \int_{0}^{\infty} d \alpha \int_{0}^{\infty} d \beta \int_{-\infty}^{\infty} \int_{-\infty}^{\infty}\left[\beta z F_{1}(\xi, \eta) \cos \alpha(x-\xi) \sin \beta(y-\eta)\right. \\
& +\left(1-\frac{\beta^{2}}{\gamma} z\right) F_{2}(\xi, \eta) \cos \alpha(x-\xi) \cos \beta(y-\eta) \\
& \left.+{ }_{\gamma}^{\alpha \beta} z F_{3}(\xi, \eta) \sin \alpha(x-\xi) \sin \beta(y-\eta)\right] e^{-\gamma z} d \xi d \eta, \\
& \widehat{z x}=\frac{1}{\pi^{2}} \int_{0}^{\infty} d \alpha \int_{0}^{\infty} d \beta \int_{-\infty}^{\infty} \int_{-\infty}^{\infty}\left[\alpha z F_{1}(\xi, \eta) \sin \alpha(x-\xi) \cos \beta(y-\eta)\right. \\
& +{ }_{\gamma}^{\alpha \beta} z F_{2}(\xi, \eta) \sin \alpha(x-\xi) \sin \beta(y-\eta) \\
& \left.+\left(1-\frac{\alpha^{2}}{\gamma} z\right) F_{3}(\xi, \eta) \cos \alpha(x-\xi) \cos \beta(y-\eta)\right] e^{-\gamma z} d \xi d \eta, \\
& \widehat{x x}=\frac{1}{\pi^{2}} \int_{0}^{\infty} d \alpha \int_{0}^{\infty} d \beta \int_{-\infty}^{\infty} \int_{-\infty}^{\infty}\left[\frac{\alpha^{2}}{\gamma^{2}}\left(1+2 \sigma \frac{\beta^{2}}{\alpha^{2}}-\gamma z\right) F_{1}(\xi, \eta) \cos \alpha(x-\xi)\right. \\
& \times \cos \beta(y-\eta)+\frac{\beta}{\gamma^{2}}\left(2 \sigma^{\frac{\beta^{2}}{\gamma}}-\alpha^{2} z\right) F_{2}(\xi, \eta) \cos \alpha(x-\xi) \sin \beta(y-\eta) \\
& \left.+{ }_{\gamma}^{2 \alpha}\left(1+\sigma \frac{\beta^{2}}{\gamma^{2}}-\frac{\alpha^{2}}{2 \gamma} z\right) F_{3}(\xi, \eta) \sin \alpha(x-\xi) \cos \beta(y-\eta)\right] e^{-r z} d \xi d \eta,
\end{aligned}
$$




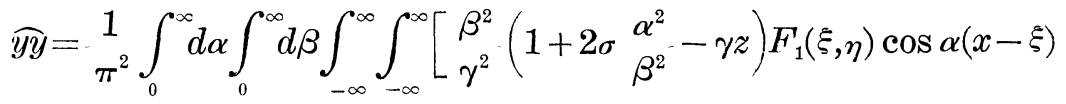

$$
\begin{aligned}
& \times \cos \beta(y-\eta)+\frac{2 \beta}{\gamma}\left(1+\sigma \frac{\alpha^{2}}{\gamma^{2}}-\frac{\beta^{2}}{2 \gamma} z\right) F_{2}(\xi, \eta) \cos \alpha(x-\xi) \sin \beta(y-\eta) \\
& \left.+\frac{\alpha}{\gamma^{2}}\left(2 \sigma \frac{\alpha^{2}}{\gamma}-\beta^{2} z\right) F_{3}(\xi, \eta) \sin \alpha(x-\xi) \cos \beta(y-\eta)\right] e^{-\gamma z} d \xi d \eta, \\
& \widehat{x y}=\frac{1}{\pi^{2}} \int_{0}^{\infty} d \alpha \int_{0}^{\infty} d \beta \int_{-\infty}^{\infty} \int_{-\infty}^{\infty}\left[-\frac{\alpha \beta}{\gamma^{2}}(1-2 \sigma-\gamma z) F_{1}(\xi, \eta) \sin \alpha(x-\xi)\right. \\
& \times \sin \beta(y-\eta)+\frac{\alpha}{\gamma}-\left(1-2 \sigma \frac{\beta^{2}}{\gamma^{2}}-\frac{\beta^{2}}{\gamma} z\right) F_{2}(\xi, \eta) \sin \alpha(x-\xi) \cos \beta(y-\eta) \\
& \left.+{ }_{\gamma}^{\beta}\left(1-2 \sigma \frac{\alpha^{2}}{\gamma^{2}}-\frac{\alpha^{2}}{\gamma} z\right) F_{3}(\xi, \eta) \cos \alpha(x-\xi) \sin \beta(y-\eta)\right] e^{-\gamma z} d \xi d \eta ; \\
& u=\frac{1}{2 \pi^{2} \mu} \int_{0}^{\infty} d \alpha \int_{0}^{\infty} d \beta \int_{-\infty}^{\infty} \int_{-\infty}^{\infty}\left[\begin{array}{l}
\alpha \\
\gamma^{2}
\end{array}(1-2 \sigma-\gamma z) F_{1}(\xi, \eta) \sin \alpha(x-\xi)\right. \\
& \times \cos \beta(y-\eta)-\frac{\alpha \beta}{\gamma^{3}}(2 \sigma+\gamma z) F_{2}(\xi, \eta) \sin \alpha(x-\xi) \sin \beta(y-\eta) \\
& \left.-\frac{1}{\gamma}\left\{2-\frac{\alpha^{2}}{\gamma^{2}}(2 \sigma+\gamma z)\right\} F_{3}(\xi, \eta) \cos \alpha(x-\xi) \cos \beta(y-\eta)\right] e^{-\gamma z} d \xi d \eta, \\
& v=\frac{1}{2 \pi^{2} \mu} \int_{0}^{\infty} d \alpha \int_{0}^{\infty} d \beta \int_{-\infty}^{\infty} \int_{-\infty}^{\infty}\left[\begin{array}{l}
\beta \\
\gamma^{2}
\end{array}(1-2 \sigma-\gamma z) F_{1}(\xi, \eta) \cos \alpha(x-\xi)\right. \\
& \times \sin \beta(y-\eta)-\frac{1}{\gamma}\left\{2-\frac{\beta^{2}}{\gamma^{2}}(2 \sigma+\gamma z)\right\} F_{2}(\xi, \eta) \cos \alpha(x-\xi) \cos \beta(y-\eta) \\
& \left.-\frac{\alpha \beta}{\gamma^{3}}(2 \sigma+\gamma z) F_{3}(\xi, \eta) \sin \alpha(x-\xi) \sin \beta(y-\eta)\right] e^{-\gamma z} d \xi d \eta, \\
& w=\frac{1}{2 \pi^{2} \mu} \int_{0}^{\infty} d \alpha \int_{0}^{\infty} d \beta \int_{-\infty}^{\infty} \int_{-\infty}^{\infty}\left[-\frac{1}{\gamma}\{2(1-\sigma)+\gamma z\} F_{1}(\xi, \eta) \cos \alpha(x-\xi)\right. \\
& \times \cos \beta(y-\eta)-\frac{\beta}{\gamma^{2}}(1-2 \sigma+\gamma z) F_{2}(\xi, \eta) \cos \alpha(x-\xi) \sin \beta(y-\eta) \\
& \left.-\frac{\alpha}{\gamma^{2}}(1-2 \sigma+\gamma z) F_{3}(\xi, \eta) \sin \alpha(x-\xi) \cos \beta(y-\eta)\right] e^{-\gamma z} d \xi d \eta .
\end{aligned}
$$

Applications of the above general solution, and numerical evaluations thereof, will be given in the succeeding paper.

\section{References}

1) K. Terazawa: Bulletin of the Faculty of Science, Tokyo University, 37 (1916).

2) A. E. H. Love: Philosophical Transactions, London, A., 228 (1929).

3) B. Tanimoto: Bulletin of the Earthquake Research Institute, Tokyo University, 26 (1948).

4) B. Tanimoto: Journal of the Shinshu University, 4 (1954).

5) B. Tanimoto: Transactions of the Japan Society of Civil Engineers, No. 24 (April, 1955).

6) Love: Elasticity, 4th ed., p. 38. 